\title{
Fabrication and Assessment of 3D Printed Anatomical Models of the Lower Limb for Anatomical Teaching and Femoral Vessel Access Training in Medicine
}

\author{
Michael K. O’Reilly, ${ }^{1}$ Sven Reese, ${ }^{2}$ Therese Herlihy, ${ }^{3}$ Tony Geoghegan, ${ }^{4}$ Colin P. Cantwell, ${ }^{5}$ \\ Robin N.M. Feeney, ${ }^{1}$ James F.X. Jones ${ }^{1 *}$ \\ ${ }^{1}$ Anatomy in the Biomedical Section, School of Medicine and Medical Science, University College Dublin, \\ Dublin, Ireland \\ ${ }^{2}$ Department of Veterinary Sciences, Section of Anatomy, Histology, and Embryology, Faculty of Veterinary \\ Medicine, University of Munich, Munich, Germany \\ ${ }^{3}$ Diagnostic Imaging, School of Medicine and Medical Science, University College Dublin, Dublin, Ireland \\ ${ }^{4}$ Department of Interventional Radiology, Mater Misericordiae University Hospital, Dublin, Ireland \\ ${ }^{5}$ Department of Radiology, St. Vincent's University Hospital, Dublin, Ireland
}

For centuries, cadaveric dissection has been the touchstone of anatomy education. It offers a medical student intimate access to his or her first patient. In contrast to idealized artisan anatomical models, it presents the natural variation of anatomy in fine detail. However, a new teaching construct has appeared recently in which artificial cadavers are manufactured through three-dimensional (3D) printing of patient specific radiological data sets. In this article, a simple powder based printer is made more versatile to manufacture hard bones, silicone muscles and perfusable blood vessels. The approach involves blending modern approaches (3D printing) with more ancient ones (casting and lost-wax techniques). These anatomically accurate models can augment the approach to anatomy teaching from dissection to synthesis of 3D-printed parts held together with embedded rare earth magnets. Vascular simulation is possible through application of pumps and artificial blood. The resulting arteries and veins can be cannulated and imaged with Doppler ultrasound. In some respects, 3D-printed anatomy is superior to older teaching methods because the parts are cheap, scalable, they can cover the entire age span, they can be both dissected and reassembled and the data files can be printed anywhere in the world and mass produced. Anatomical diversity can be collated as a digital repository and reprinted rather than waiting for the rare variant to appear in the dissection room. It is predicted that $3 \mathrm{D}$ printing will revolutionize anatomy when poly-material printing is perfected in the early 21st century. Anat Sci Educ 9: 71-79. () 2015 American Association of Anatomists.

Key words: gross anatomy education; radiology education; residency training; 3D imaging techniques; 3D printing; rapid prototyping; ultrasound simulation; low-fidelity simulation models; anatomical sciences; digital anatomy

\footnotetext{
${ }^{*}$ Correspondence to: Prof. James F.X. Jones, Anatomy, School of Medicine and Medical Science, University College Dublin, Dublin 4, Ireland. E-mail: James.jones@ucd.ie

Received 28 January 2015; Revised 27 March 2015; Accepted 13 April 2015.

Published online 24 June 2015 in Wiley Online Library (wileyonlinelibrary.com). DOI 10.1002/ase.1538

(c) 2015 American Association of Anatomists
}

\section{INTRODUCTION}

The application of three-dimensional (3D) printing technology to anatomy has been recently highlighted in the Anatomical Sciences Education journal by $\mathrm{Li}$ et al. (2012) and McMenamin et al. (2014), as well as in other journals by Rengier et al. (2010), Torres et al. (2011), and Vaccarezza and Papa (2015). Rose et al. (2015) describe the use of multimaterial temporal bones for drilling/dissection and Watson (2014) advocates the outsourcing of printing models (hepatic 
vessels) to commercial companies. The study by McMenamin et al. (2014) in particular, demonstrates the potential value of the $3 \mathrm{D}$ printing approach to the teaching of anatomy. Prosected specimens can be transformed into 3D color models and economically mass manufactured at various scales. As the authors were using a $\mathrm{Z}$ Corp powder printer (ZPrinter ${ }^{\circledR} 650$; 3D Systems Corp., Rock Hill, SC) the resulting models exhibited a high Shore hardness and brittleness. But the authors predicted that future soft dissectable models will involve using multi-material printers (such as the Stratsys Objet Connex Multi-Material printers; Stratasys Ltd., Eden Prairie, MN). Unfortunately, this technology is prohibitively expensive and the photopolymer resins are also costly.

The present study presents the results of some of experiments in $3 \mathrm{D}$ printing in anatomy using a simple powder $\mathrm{Z}$ Corp printer (ZPrinter ${ }^{\circledR} 250$, 3D Systems Corp., Rock Hill, SC). The authors have increased the versatility of this powder printer by creating hard molds for soft silicone muscles and applying the "lost-wax casting technique" to hard powder cores for soft blood vessels. The introduction of ultrasound to anatomy teaching can vividly impress upon students the relation between grayscale sonographic images and the corresponding anatomy (Moscova et al., 2015). However, some caution must be exercised with the sonographer's terms for anatomical landmarks (Hall et al., 2015). Although ultrasound equipment is becoming cheaper and a more common imaging modality for the dissection room, circulatory flow is seldom re-established in the formaldehyde fixed cadaver and therefore Doppler imaging is impossible. In this article, a description is provided of local manufacture of anatomically accurate blood vessels and a method for perfusing these with artificial blood. Vascular models of the inguinal region were created in order to teach anatomy to medical students and cannulation methods to interventional radiologists. A study by Seto et al. (2010) has shown that the more experience an operator has with real time ultrasound the greater the chance of success of femoral artery access (especially in cases of high branching of the femoral artery). Therefore, another aim of the present study was to capture these anatomical variants in model form.

\section{METHODS}

Approval from the local human research ethics committee was obtained to use fully anonymized patient CT data sets and to survey users of the 3D-printed models with voluntary questionnaires.

\section{Construction of Lower Limb Model}

The printed lower limb was based on freely available anatomically accurate 3D model files from the BodyWorks3D website (Center for Life Science, 2013) as object (.obj) files (Mitsuhashi et al., 2009). Object files were downloaded for each part of the right lower limb model, limited to bones from the femur to the phalanges and the muscles and tendons of the posterior compartment of the leg.

These files were opened in a free mesh processing software MeshLab, version 1.3.3, (Visual Computing Lab, ISTI-CNR, Pisa, Italy). Using this software, the number of faces/vertices were reduced in each model by applying a quadric edge collapse decimation filter, set to $30 \%$ of the original number of faces/vertices, thus reducing the size and complexity of each rendered model. This allows for smoother editing and processing in the printing software without compromising topographical detail. These filtered files were then exported as stereolithography files (.stl), the ubiquitous and universal file standard in 3D printing.

The files containing the data for the posterior compartment muscles were imported into the software Tinkercad (Autodesk Inc., San Francisco, CA), a free cloud based graphical user interface computer aided design software package that permits (.stl) file manipulation and editing. The 3D models of the muscles were placed in a virtual box large enough to contain their entire structure and a Boolean subtraction function removed the external muscle shape from the inner volume of the box thereby creating a negative mold of the muscle.

The muscle mold (.stl) files were then opened in $\mathrm{Z} \mathrm{Edit}{ }^{\mathrm{TM}}$ Pro, (3D Systems Corp., Rock Hill, SC) a proprietary mesh editing and 3D printing preparation software package. This software fixed mesh errors and allowed the mold to be cut in half and connector pins and drain holes to be added.

The bone files were imported directly into $\mathrm{Z} \mathrm{Edit}{ }^{\mathrm{TM}}$ Pro without the Tinkercad step as described above. Using the drain hole function cylindrical cavities were placed into the models at the origin and insertion of the posterior compartment muscles and also the bony articulations. The cavity size was dependent on the size of miniature disc magnet to be inserted. The files were then opened in Z Print, (3D Systems Corp., Rock Hill, SC) and scaled to 1: 2 to reduce model cost and to allow all parts to be manufactured in one print run. $Z$ Print then sent the finalized models to our Binder Jetting (BJ) 3D printer, ZPrinter ${ }^{\circledR} 250$, (3D Systems Corp., Rock Hill, SC). This printer is a 64 color composite 3D printer with a 400 micron minimum feature size and layer thickness of 100 microns. It has a build volume of $236 \times 185 \times$ $127 \mathrm{~mm}$ and a printing speed of $20 \mathrm{~mm}$ per hour. The print material, ZP 151, (3D Systems Corp., Rock Hill, SC) is a proprietary formula based on calcium sulfate dihydrate and other materials that are trade secrets.

The total print and drying time for the entire model and molds was 7.5 hours. Once the bones and muscle molds were depowdered they were baked at $60^{\circ} \mathrm{C}$ in an oven, (WON-32; Daihan Scientific, Gangwon-do, Korea) for 2 hours to complete the binding process. The bones and muscle molds were then finished using ZBond, (3D Systems Corp., Rock Hill, SC), a cyanoacrylate based infiltrant designed to rapidly strengthen composite 3D-printed models.

The materials to create the muscles were commercially available room temperature vulcanization (RTV) addition/ platinum cure high tear strength silicones, Dragon Skin Series 2A and Eco-Flex 00-50A, (Smooth-on Inc., Macungie, PA). The A rating is a measure of hardness using the Shore Hardness Scale, based on the induration caused by a $1.4 \mathrm{~mm}$ steel rod with a $0.79 \mathrm{~mm}$ diameter $35^{\circ} \mathrm{C}$ truncated cone applying $8.064 \mathrm{~N}$ of pressure (Mix and Giacomin, 2011). All our samples were subjected to negative pressure $(-30 \mathrm{~mm} \mathrm{Hg})$ to remove air bubbles for two minutes in a vacuum chamber.

Each muscle mold was prepared by spraying Ease Release $^{\circledR}$ 200, (Smooth-on Inc., Macungie, PA), a petroleum based silicone release agent that would facilitate demoulding. The tendinous insertion and origin were molded using EcoFlex 00-50A. Ten grams of iron filings (Sigma-Aldrich Ireland Ltd. Arklow, Ireland) were added to $20 \mathrm{~mL}$ of Eco-Flex 00$50 \mathrm{~A}$ in order to make the silicone ferromagnetic. The belly of the muscle was formed with Dragon Skin ${ }^{\circledR}$ No. 20A, colored with blood red Silc Pig ${ }^{\circledR}$ (Smooth-on Inc., Macungie, PA). As 

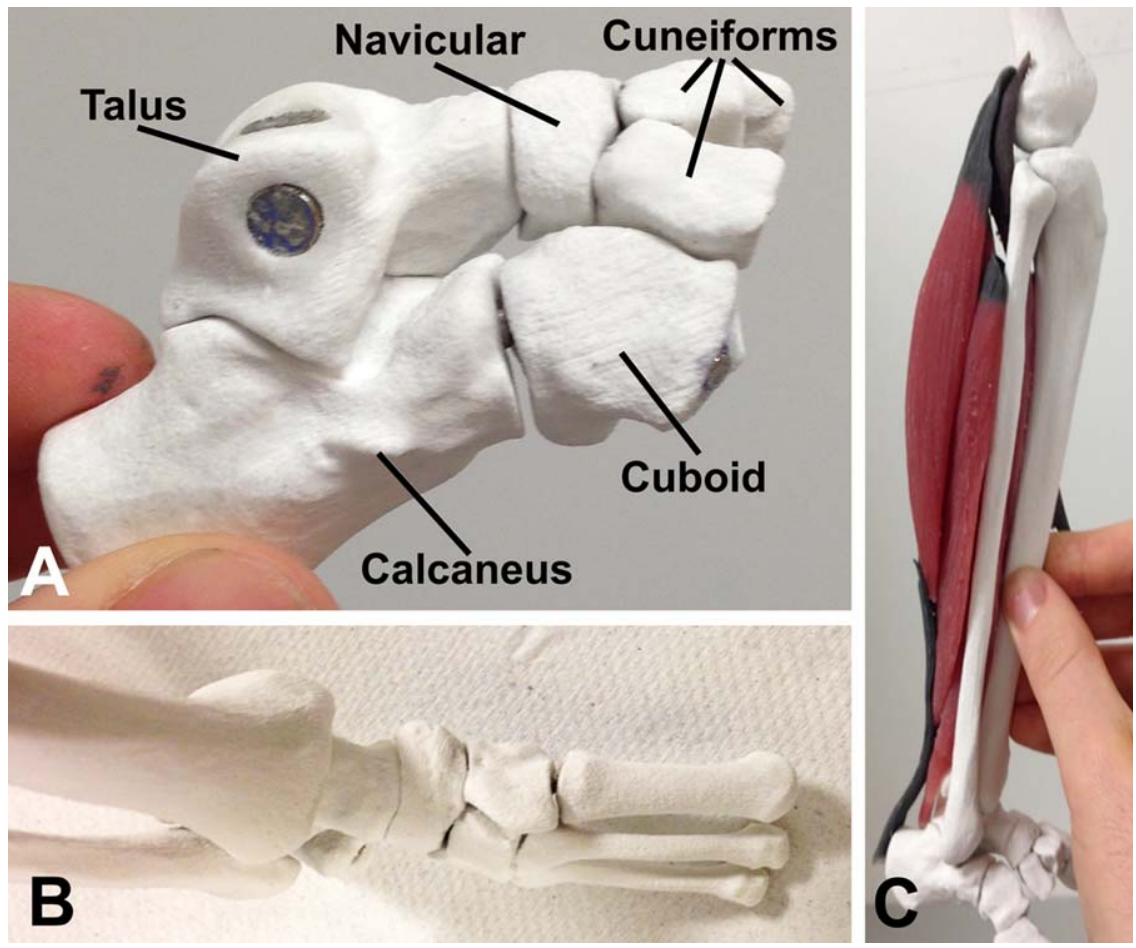

\section{Figure 1.}

Lower limb 3D-printed anatomical model. This 22-part model of the leg and foot was produced in one print run of the Z Corp system. A, shows small bones of the foot held together with rare earth magnets; B, shows dorsum of foot with tibia and fibula attached; and C, shows the silicone muscles of the calf which are attached to bones via magnetized tendons.

both Eco-Flex and Dragon Skin are platinum catalyzed silicones they do not exhibit cure inhibition when mixed; full cure was obtained by heating the silicones in an oven at $60^{\circ} \mathrm{C}$ for one hour.

The bones were then assembled and Neodymium rare earth magnets (First4Magnets, Tuxford, Nottinghamshire, UK) glued into the preformed cylindrical cavities at the bony articulations and the muscles origins and insertions (Fig. 1). The $3 \mathrm{~mm} \times 2 \mathrm{~mm}$ (diameter $\times$ height) magnet used in the joints between the cuneiforms and the metatarsals was able to support 270 grams of weight and the two $6 \mathrm{~mm} \times 4 \mathrm{~mm}$ magnets in the knee joint were designed to support $1.2 \mathrm{~kg}$ each. This enabled the model to be suspended by the knee joint without risk of it falling apart. Each model required 34 magnets and $10 \mathrm{~g}$ of iron filings in total.

\section{Limb Model Assessment}

An electronic message was circulated to our Graduate Entry Medicine Year 1 class inviting them to a supplementary practical session on lower limb anatomy to explore the use of 3D printing in anatomical education. This class had just completed their anatomy modules on limb anatomy. Of the 105 students invited 22 students attended on the day. When they arrived they were randomly divided into two groups, a 3Dprinted model group and a dissection group, using Random.org (Randomness and Integrity Services Ltd., Dublin, Ireland), a random number generator. A 10-question formative assessment was performed to compare baseline anatomical knowledge of the lower limb of the two groups. The tests were performed using the Qwizdom ${ }^{\circledR}$ Student Response Interactive System, (Qwizdom, Puyallup, WA).

Subsequently the 3D-printed model group was led to our clinical skills laboratory where three unassembled lower limb models were available. The dissection group remained in the dissection laboratory. Each group was given an identical set of objectives to accomplish regarding the calf muscles and the bones of the lower limb. They had access to an anatomy demonstrator and anatomy atlases for the duration of the one hour practical.

At the end of the practical, after a 15 -minute break, both groups reconvened in the dissection room where a further 28-question assessment was performed specifically on the material covered in the practical session guidelines using the Qwizdom system.

Following the assessment a survey was provided to the students which included questions about their preferred learning methods. This survey used a six-point Likert scale to quantify responses.

\section{Construction of Femoral Artery Access Model}

The vascular 3D models were created with the software Mimics $^{\mathrm{TM}}$, version 16.0 (Materialise, Leuven, Belgium) using segmentation algorithms applied to anonymized patient computed tomography data sets or by downloading free vascular models from the BodyWorks3D website (Center for Life Science, 2013). Calcium sulphate dihydrate, the main 

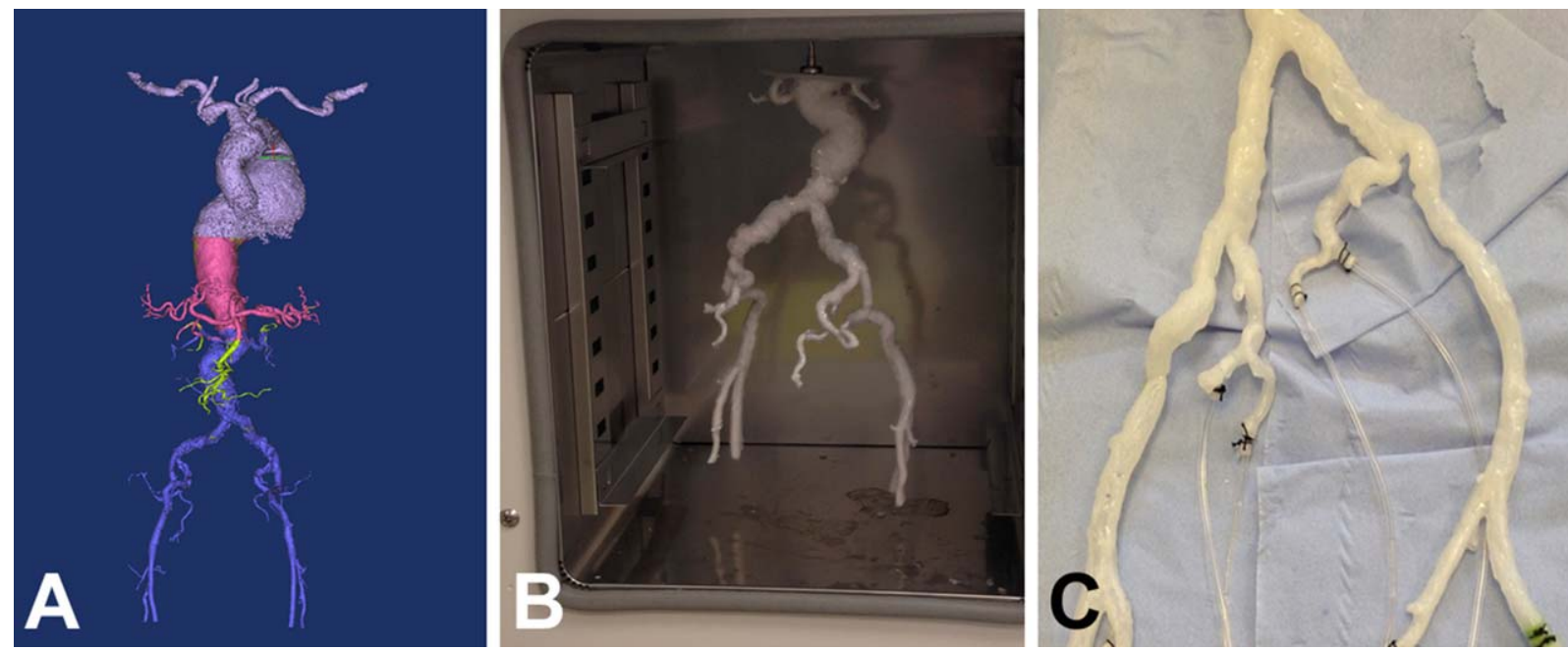

\section{Figure 2.}

An aneurysmal atherosclerotic circulation. A, The segmented circulation from subclavian arteries to femoral arteries (derived from a CT angiogram); B, A silicon coated powder printed vasculature in a drying oven; C, Following removal of the powder core the silicone phantom remains intact and can be perfused.

component of the ZP151 powder is soluble in water at $0.24 \mathrm{~g} / 100 \mathrm{ml}$ at $20^{\circ} \mathrm{C}$ (American Chemical Society, 2006). The process of producing hollow silicone arterial vessels is similar to the lost-wax casting method. The ZP 151 model was coated with a fine mist of Epsom salts $(44 \%$ w/v) with a small amount of pink food coloring (Tesco PLC, Hertfordshire, UK) to visualize which areas of the model had been adequately coated. After this coat of Epsom salt the model was baked in a gravity oven at $60^{\circ} \mathrm{C}$ for 30 minutes and the whole process repeated three times.

Once the models were dry, the Dragon Skin series silicon rubbers (No 20A) two-component room temperature vulcanization silicone (series 2A, Smooth-on Inc., Easton, PA) addition cure silicone was used to create the muscular tunic of the vessel. The model was then placed in a gravity oven at $60^{\circ} \mathrm{C}$ for 12 minutes to accelerate the curing process. This also had the added benefit of reducing dripping and accumulation of silicone that can result in a nonuniform wall thickness. As each coat was very thin, 0.3 to $0.5 \mathrm{~mm}$, eight minutes was sufficient to reach a state where the silicone had polymerized into a solid layer. This step was repeated four times and the model then baked for a further two hours to complete the curing process. The powder core of the model was removed by flushing with hot tap water and then placed in a sealed 5-liter beaker of distilled water and placed in a gravity oven at $80^{\circ} \mathrm{C}$ for 8 to 10 hours. This resulted in a patent, hollow replica of the arterial segmented lumen with a 1 to $2 \mathrm{~mm}$ translucent silicone wall (Fig. 2). A fused deposition Ultimaker Original 3D printer (Ultimaker B.V., Geldermalsen, The Netherlands), was used to produce bespoke connectors for our arterial models (in polylactic acid). They were modeled on the arteries diameters thus avoiding distortion of the model when applied. The connectors were secured in place using 1-0 silk surgical suture. Using the MIMICS $^{\mathrm{TM}}$ segmentation algorithm, the threshold Hounsfield values were set from 300 to 1,200 to isolate bone and from 30 to 100 to segment skin. The anterior surface of the segmented skin model was extruded uniformly by $5 \mathrm{~mm}$ to ensure it was robust. The resultant (.stl) files were processed with ZEdit pro
(3D Systems Corp., Rock Hill, SC) and printed with the Z250 BJ 3D printer (3D Systems Corp., Rock Hill, SC). The mask of the patient's skin was between 1.5 and $2.3 \mathrm{~mm}$ in depth, in keeping with described reference ranges (Akkus et al., 2012). Dragon Skin (No. 10A; Smooth-on Inc., Easton, PA) was utilized as a tissue mimicking material as it has been previously used in other medical simulators and has a similar Poisson's ratio (0.25) to skin (0.30) (Agache et al., 1980; Miller et al., 2013). The area of the skin mould was measured and the desired silicone skin thickness set at $2 \mathrm{~mm}$. Tissue mimicking material was produced by mixing ballistic gelatin (Dr. Oetker Ireland Ltd., Ballymount, Dublin, Ireland) and corn starch. The femoral artery/vein simulator was perfused with artificial blood driven by a peristaltic pump, model 505S, (Watson-Marlow Ltd., Falmouth, Cornwall, UK). The artificial blood consisted of distilled water (1 1), glycerol (12 g\%), sephadex beads (0.4 g\%; diameter range 50-100 microns; Sigma-Aldrich Ireland Ltd. Arklow, Ireland) and blood coloring liquid (1gram\%), Adam,Rouilly Ltd., Sittingbourne, Kent, UK). The arterial perfusion pressure was set between 80 and $100 \mathrm{~mm} \mathrm{Hg}$ by adjusting a resistor (Hoffman clamp) on the outflow tubing and measured by noting the height of a column of water.

\section{Assessment of Femoral Artery Access Model}

To examine the utility of our models for learning ultrasoundguided arterial puncture techniques they were presented to the entire cohort of Irish first year radiology specialist registrars $(n=19)$ and two consultant interventional radiologists. Each year there is a training day run by the Faculty of Radiologists for the specialty of interventional radiology. Currently the Ultrasound Vascular Access Slab, (part 60531, Limbs \& Things Ltd., Bristol, UK), a low-fidelity ultrasound access trainer is used for this session. Each trainee had hands on time with the faculty model and one of our two models. They were then asked to fill out a survey with a six-point Likert scale for 3D model and also on preferred learning 


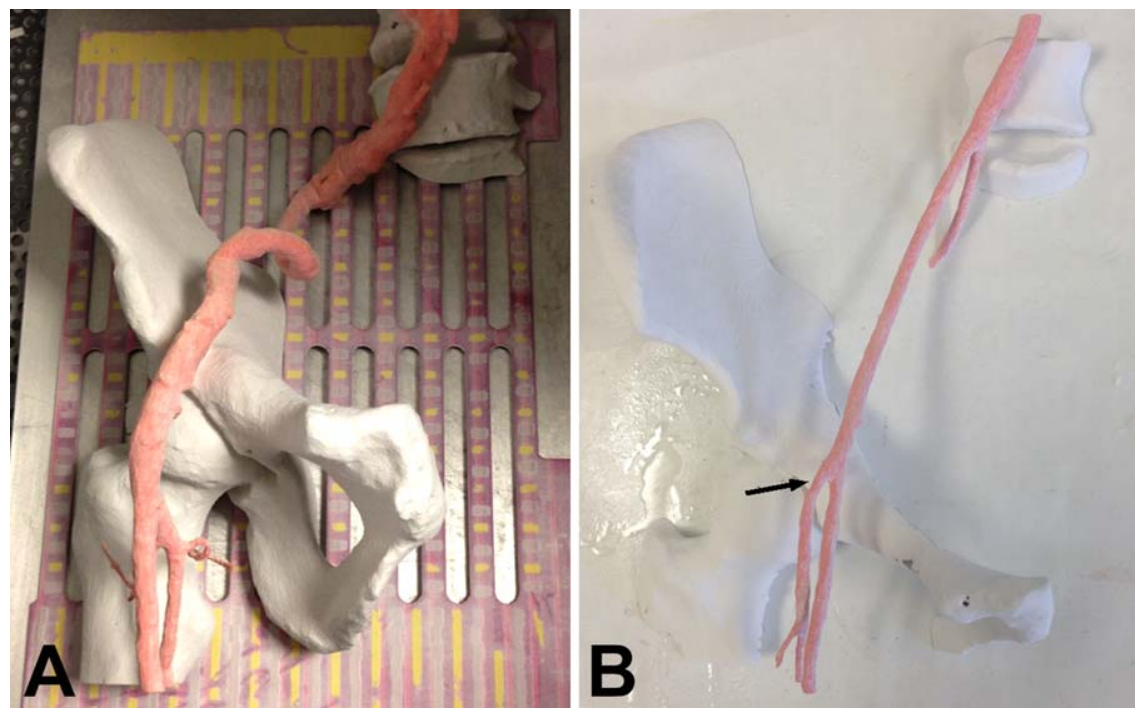

\section{Figure 3.}

Patient specific vascular anatomy. A, shows the tortuous vascular anatomy of an elderly female with atherosclerosis. This is how the color printed parts appear when removed from the machine; $\mathbf{B}$, shows a more healthy vasculature but with a high origin of profunda femoris artery (black arrow).

modalities in interventional radiology. There was also an open section for comments.

\section{Statistical Analysis}

Results were analyzed using SPSS software (version 20.0, IBM Corp., Armonk, NY). The medical student data was analyzed with an unpaired Student's $t$ test. A five-item questionnaire for radiologists' opinion of the vascular model was tested for internal consistency with Cronbach's alpha and gender differences with Tau B. The criterion for statistical significance was $P \leq 0.05$.

\section{RESULTS}

\section{Construction of Lower Limb Model}

A 22-part scale model (1:3) of the lower limb and posterior compartment musculature was successfully manufactured using the BodyParts3D data set (Fig. 1). The model consisted of all the bones of the lower limb excluding the patella to the level of the metatarsal bones. The bones were connected at their articulations using rare earth magnets that permitted the student to both assemble and disassemble the model. Muscle attachments were also reversible as the silicone replicas had black ferromagnetic tendons (Fig. 1).

\section{Limb Model Assessment}

Twenty two students $(21.6 \%$ of the class $)$ participated. Results were analyzed using SPSS statistical package, version 20 (IBM Corp., Armonk, NY) and unpaired Student's $t$-test. In the formative test, no difference $(P=0.63)$ was found between the initial baseline knowledge of the 3D-printed model group $(49.1 \%)$ and the dissection room (DR) group $(48.2 \%)$. Following the one hour practical session, the 3Dprinted model group averaged $50 \%$ and the DR group
$45.5 \%(P=0.46)$. The 3D-printed group strongly agreed that the $3 \mathrm{D}$ model is a learning tool they would like to use in anatomy $(90.6 \%)$ and both 3D-printed model $(90.6 \%)$ and DR $(83.3 \%)$ groups agreed their learning was enhanced using interactive models.

\section{Construction of Femoral Artery Access Model}

Two full-scale vascular models were produced. Model 1 utilized a diseased, dilated arterial system from an elderly patient with heavy atherosclerotic calcification (Fig. 3). Model 2 was derived from a healthy 50-year-old female; her vascular anatomy displayed an early branching of profunda

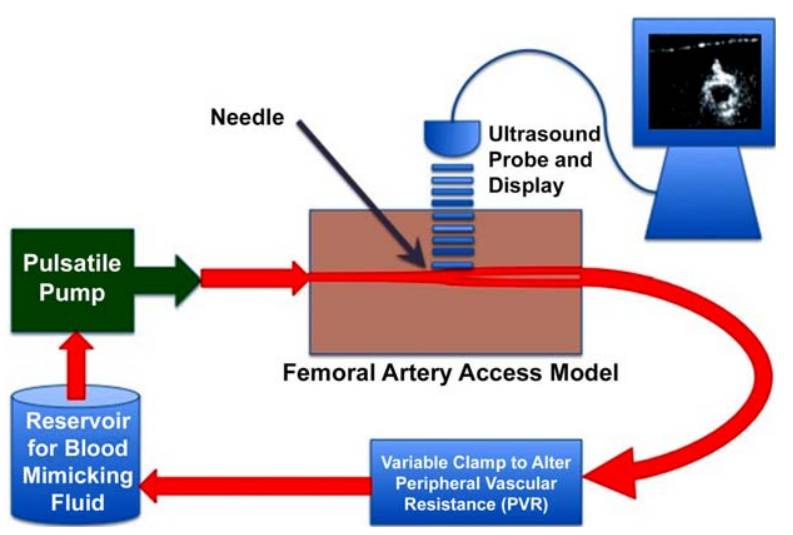

\section{Figure 4.}

Schematic representation of experimental procedure for performing Doppler ultrasound on perfused 3D-printed vessel. A peristaltic pump drives pulsatile flow at arterial pressure which can be detected with an ultrasound probe. This facilitates arterial cannulation via percutaneous needle insertion. 

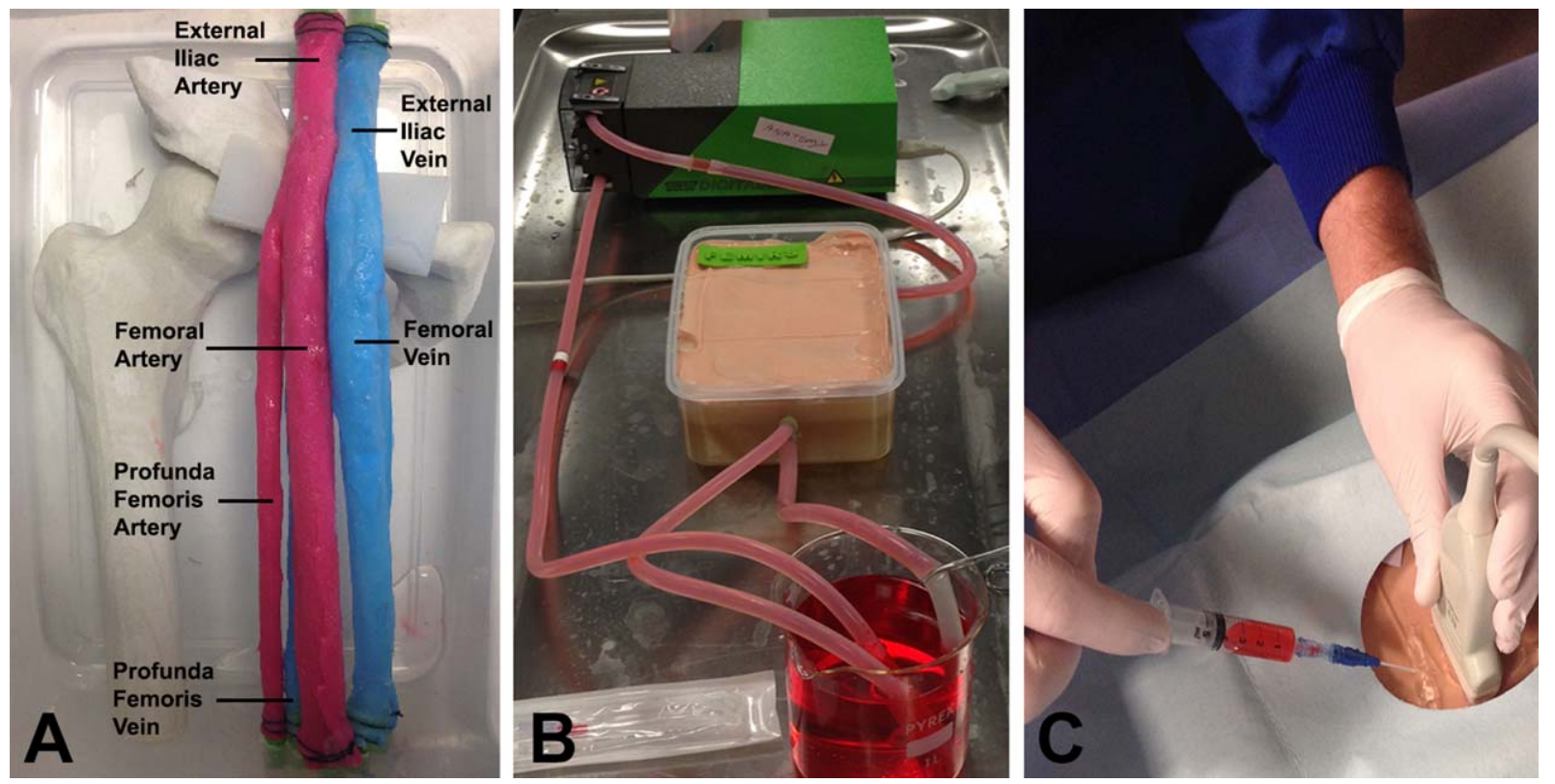

\section{Figure 5 .}

Femoral artery and vein model. This model of a patient's inguinal region contains skin, adipose tissue, bone, pulsatile arteries and smooth venous flow; A, shows the early stage of the model construction with bony parts and silicone vessels and before the tissue mimicking material has been added; $\mathbf{B}$ shows the complete model with tissue and skin layer. A simple peristaltic pump driven at 70 revolutions per minute provides the motive force for the circulation of artificial blood; C, illustrates ultrasound guided arterial puncture and withdrawal of artificial blood.

femoris artery from the femoral artery, a normal but important anatomical variant (Fig. 3).

\section{Assessment of Femoral Artery Access Model}

Nineteen trainees took part in the survey of these vascular models which were perfused and imaged with ultrasound
(Fig. 4). The average age of the cohort was 29.5 years and there was an equal representation of gender. The results showed that the trainees preferred our model compared to the commercially available simulator with an overall rating of 5.1/6. A model was created that looked realistic during ultrasound imaging (5.2/6) and had haptic characteristics that would be expected in a real patient experience including a palpable pulse (4.3/6). Our overall aim of creating a model
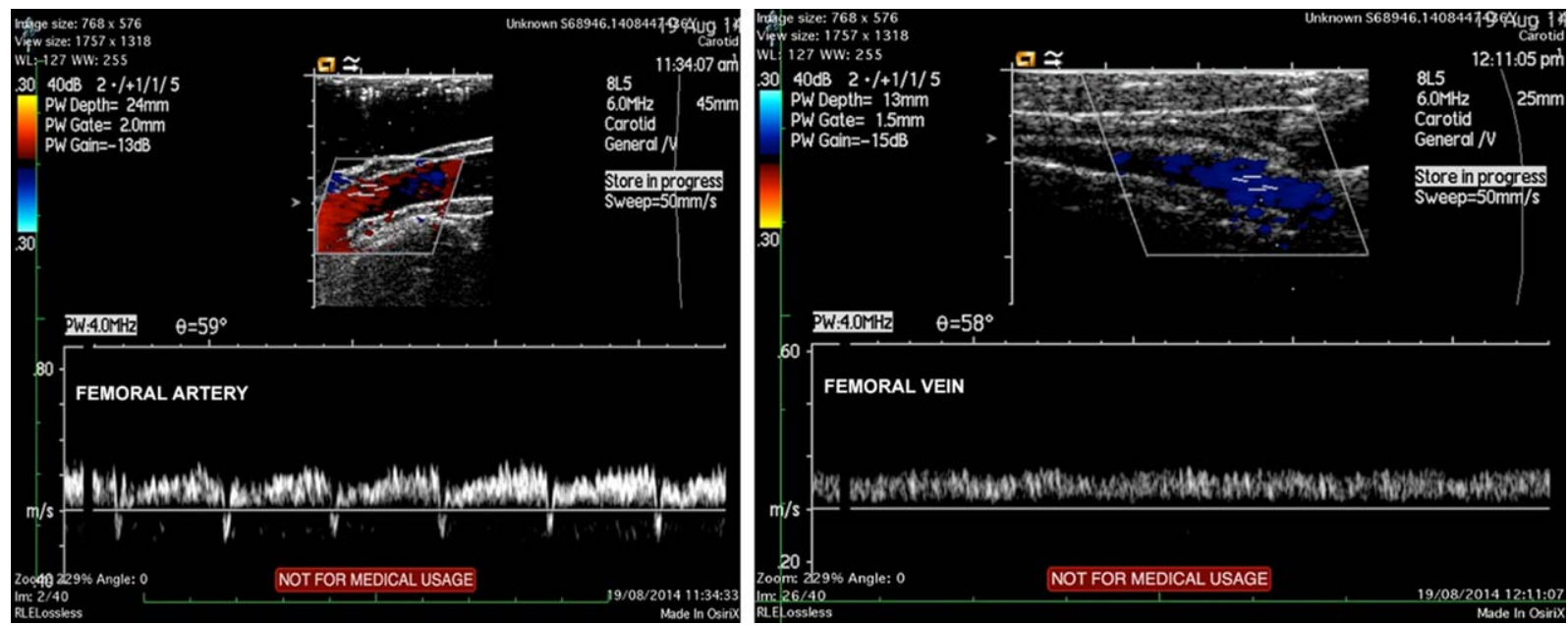

\section{Figure 6.}

Longitudinal Doppler ultrasound imaging of the femoral artery (left panel) and femoral vein (right panel). The color coding indicates the direction of blood travel with the arterial wave front being centrifugal and the smooth venous flow centripetal. 

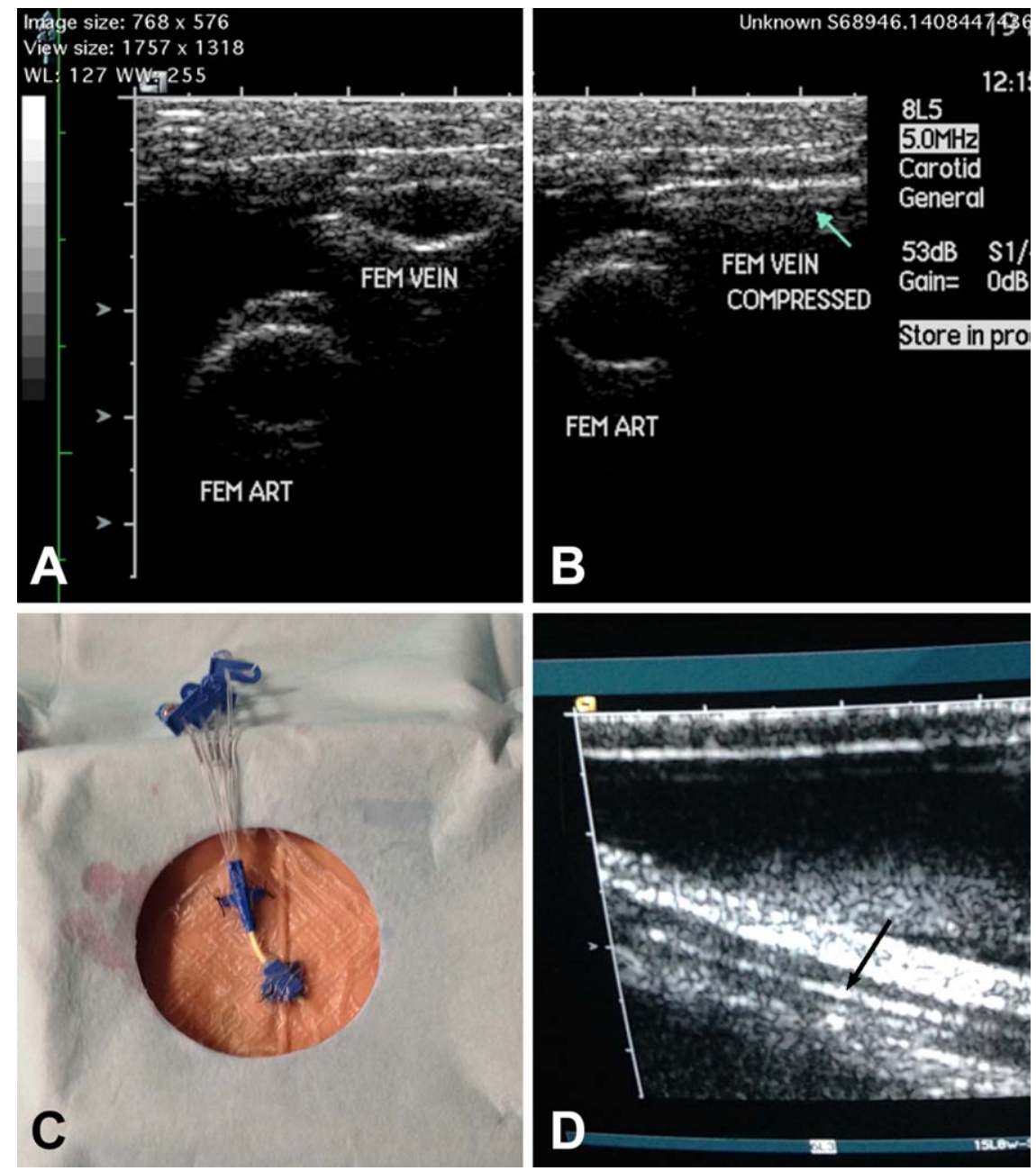

\section{Figure 7.}

Imaging femoral artery (FEM ART) and femoral vein (FEM VEIN) with ultrasonography. A and B, show a compressive force placed on the skin overlying the femoral vessels that leads to its selective venous collapse. This is a standard technique used to distinguish femoral artery and vein when interventional radiological access is required; $\mathrm{C}$, the vessels can be cannulated percutaneously with a venous access catheter; and $\mathrm{D}$, the cannula (black arrow) is imaged within the vessel lumen.

that mimics a real patient encounter was also accomplished with a rating of $4.5 / 6$. A sample of the questionnaire is included in the supplementary material. The composite score for the five items was $4.6 / 6$ and its internal consistency was tested with Cronbach's alpha and was found to be 0.74 . The average scores of female and male participants for the five items were positively and significantly correlated (Tau $\mathrm{B}=0.8$; two tailed $P=0.05$ ) indicating that there was no gender bias in the response patterns.

In response to the qualitative feedback received, improvements were made to model including the near field echogenicity of the tissue mimicking material (TMM), an anatomically accurate, compressible venous system and inclusion of materials that do not degrade with time (e.g. gelatin slowly liquefies).

The near field echogenicity was improved by simply increasing the concentration of cornstarch in a subcutaneous layer to $18 \mathrm{~g} / \mathrm{l}$ and setting the TMM in layers as opposed to a single pour in the original model (Gerstenmaier et al.,
2013). Gelatin has a finite lifespan, even when refrigerated and stored in an airtight container. Florid fungus formation and liquefaction of the gelatin formed after week 3 of storage and by week 4 most models were unusable. Using gel wax (FullMoons Cauldron, Berkshire, UK) previously described in use of a model breast biopsy phantom (Vieira et al., 2013), the model evolved to be odorless, degradation free and with similar acoustic properties to human tissue. This gel wax feels like subcutaneous fat and has a density of $0.81 \mathrm{~g} / \mathrm{cm}^{3}$ and a melting point of $61.4^{\circ} \mathrm{C}$. Cornstarch can also be mixed with the wax to adjust its echogenicity, at similar concentrations to those used with gelatin previously.

The final model was a high-fidelity, low-cost ultrasound phantom. It had a palpable pulse, similar Doppler signals to in vivo arterial and venous waveforms and vessels that can be cannulated many times before repair is needed. It also demonstrated similar ultrasound properties to real tissue with a thin walled collapsible femoral vein and pulsatile pressurized thicker walled femoral artery (Figs. 5-7). 


\section{DISCUSSION}

Rapid prototyping has been reported to be of benefit for surgical planning and education in cardiac pediatric surgery (Costello et al., 2014, 2015), plastic surgery (Gerstle et al., 2014), orthodontics (Groth et al., 2014), and neurovascular surgery (Khan et al., 2014). The present research describes methods to increase the versatility of a single material printer by printing molds for silicone muscles and vascular cores for perfusable blood vessels. The freely available BodyParts3D resource (Center for Life Science, 2013) represents a particularly valuable repository for teachers of anatomy as it contains thousands of anatomically accurate body parts (bones, muscles, blood vessels, etc). Regional anatomy can be constructed by combining the various printable files into a single model. These can be supplemented with color, text, barcodes, or quick response $(\mathrm{QR})$ codes. As a further refinement, the models can be made more physiological by introducing pulsatile blood flow at physiological pressures. Although not described in this paper the silicone muscles can also be made to shorten by embedding pneumatic filaments into the muscle bellies (McKibben artificial muscles). This has been reported for an anatomically accurate silicone heart which generates a realistic systole (Roche et al., 2014).

Both the lower limb and femoral artery models were tested on subjects for their educational value. The resulting studies were rather small as only one fifth of the medical class participated in the study and the total number of trainee interventional radiologists in Ireland is limited. There was no statistical difference between the 3D-printed model group and DR group with regard to knowledge of lower limb anatomy. This suggests that the model building is a useful adjunct to Anatomy education particularly in those departments that lack access to cadavers. The qualitative feedback obtained from both cohorts was useful and led to improvements of the models. In particular, the addition of the femoral vein with reversed blood flow and compressibility was deemed a key feature for realism and usefulness in radiological anatomy. A recent Irish study published in the journal Surgeon, demonstrated that final year medical students retention of foot and ankle anatomy was poor (Groarke et al., 2015). When 235 students were shown a normal X-ray image of the foot and ankle with six labeled bones, only $8.3 \%$ scored $6 / 6$ (the average score was 2.7/6). The findings of the present small pilot study with students are encouraging and agree with the results of other small pedagogical studies and surveys (Motoike et al., 2009; Preece et al., 2013).

Typical simulators for radiology or surgical simulation have revolved around creating models, either in the virtual world with simulated haptic feedback, manufactured crudely from unrealistic materials or from biological tissues (Vidal et al., 2009; Petrinec et al., 2011; Johnson et al., 2012; Dugo et al., 2014; Villard et al., 2014). Only recently have the first examples of 3D-printed surgical simulators been described. Urology education has been augmented with a patient specific percutaneous nephrolithotomy simulator built using a fused deposition modeling (FDM) printer and water soluble print material (Cheung et al., 2014; Turney, 2014).

\section{Limitations of the Study}

One limitation of the present study is the lack of objective evidence that practice with $3 \mathrm{D}$ femoral model increases pro- cedural skill. Simulation based education however has been shown to transfer to patient level care (McGaghie et al., 2011) and to predict the level of clinical experience of the user in central venous catheter (CVC) line placement (Dong et al., 2010). In order to validate this model as a valuable learning tool and to improve patient safety a set of guidelines for the safe placement of femoral central lines and femoral arterial access will need to be formulated in the future. Another limitation is that the femoral arterial waveform did not exhibit a sharp upstroke despite efforts to manipulate flow and pressure. However, the flow velocity profiles were clearly different in artery and vein. Despite the fact that the advantages of rapid prototyping have been employed in this study there is residual manual craft required for model completion. The models could have been printed more conveniently by a third party especially by companies that have access to expensive multi-material printers. And although the method for manufacture of silicone vessels is relatively simple and cheap it does not permit control of vessel wall thickness.

\section{CONCLUSIONS}

Both freely available printable anatomical surface models and those segmented from radiological data sets augmented the teaching resources of anatomy. The models were patient specific and interactive and made the practical component of anatomy interactive for medical students. The printed models of the leg were not inferior to cadaveric based teaching when standardized testing of anatomical knowledge was used. The 3D-printed simulators of femoral artery access were judged to be useful teaching aids and realistic. Threedimensional printing technology is expanding and becoming increasingly economical and the authors predict that it will become an essential instrument of anatomy education within the next $5-10$ years. The range of printable materials is increasing and this advance in material science will yield the range of base materials to construct the fundamental tissues of the body. In addition the advent of electrically conductive and magnetic materials will permit printed circuitry to be integrated within printable objects. Reprintable medicine may overtake regenerative medicine as pure engineering solutions can be devised for limb prosthesis without the use of stem cells. Advances in this area of anatomical research will have parallel and profound effects on the teaching of anatomy.

\section{ACKNOWLEDGMENTS}

The authors acknowledge the support of the School of Medicine and Medical Science, University College Dublin and the Faculty of Radiology, Royal College of Surgeons in Ireland.

\section{NOTES ON CONTRIBUTORS}

MICHAEL O'REILLY, M.B. B.Ch., B.A.O., M.R.C.S.I., is a specialist registrar in radiology in the Mater Misericordiae University Hospital in Dublin, Ireland. He recently (20132014) held a post as an anatomy demonstrator in the School of Medicine of University College Dublin in Dublin.

SVEN REESE, Ph.D., Dr. Med. Vet., is a senior lecturer in the Department of Veterinary Sciences of the University of Munich, Munich, Germany. He has been teaching students in anatomy und ultrasound diagnostics for 23 years and his 
research interest is in ultrasonography in veterinary anatomy teaching.

THERESE HERLIHY, B.Sc. (Radiography), M.Sc. (Medical Ultrasound), M.Sc. (Public Health), is a sonographer and lecturer in diagnostic imaging in the School of Medicine and Medical Science, University College Dublin, Dublin, Ireland. Her research and teaching is in ultrasound training and the role of ultrasound in improving health care.

TONY GEOGHEGAN, M.B., M.Sc., M.R.C.P.I., M.R.C.O.G., F.F.R. (R.C.S.I.), is a consultant interventional radiologist in the Department of Interventional Radiology at Mater Misericordiae University Hospital in Dublin, Ireland. His research interest is in interventional radiology in women's health.

COLIN P. CANTWELL, M.Sc., F.F.R. (R.C.S.I.), F.R.C.R., F.S.I.R., E.B.I.R., is a consultant interventional radiologist in the Department of Radiology at St. Vincent's University Hospital in Dublin, Ireland. He is instructor in interventional radiology at the Faculty of Radiology, Royal College of Surgeons in Ireland and his research interest is in ultrasound phantoms.

ROBIN N.M. FEENEY, Ph.D., is a lecturer in anatomy and physical anthropology in the School of Medicine and Medical Science at the University College Dublin, Dublin, Ireland. She teaches medical and allied health students and her research interest is in dental anthropology.

JAMES F.X. JONES, M.D., Ph.D., is a professor of anatomy and the Chair and Head of Anatomy in the School of Medicine and Medical Science, University College Dublin, Dublin, Ireland. He has been teaching physiology and anatomy to medical students for 25 years and his research interest is in autonomic neuroscience.

\section{LITERATURE CITED}

Agache PG, Monneur C, Leveque JL, De Rigal J 1980. Mechanical properties and Young's modulus of human skin in vivo. Arch Dermatol Res 269:221232.

Akkus O, Oguz A, Uzunlulu M, Kizilgul M 2012. Evaluation of skin and subcutaneous adipose tissue thickness for optimal insulin injection. J Diabetes Metabol 3:216.

American Chemical Society. 2006. Reagent Chemicals: Specifications and Procedures. 10th Ed. New York, NY: Oxford University Press, Inc. p 816.

Center for Life Science. 2013. BodyParts3D ${ }^{\oplus}$ Anatomography. The Database Center for Life Science. Tokyo, Japan: Life Science DB. URL: http://lifesciencedb.jp/bp3d/ [accessed February 28, 2015].

Cheung CL, Looi T, Lendvay TS, Drake JM, Farhat WA. 2014. Use of 3dimensional printing technology and silicone modeling in surgical simulation: Development and face validation in pediatric laparoscopic pyeloplasty. J Surg Educ 71:762-767.

Costello JP, Olivieri LJ, Krieger A, Thabit O, Marshall MB, Yoo SJ, Kim PC, Jonas RA, Nath DS. 2014. Utilizing three-dimensional printing technology to assess the feasibility of high-fidelity synthetic ventricular septal defect models for simulation in medical education. World J Pediatr Congenit Heart Surg 5: 421-426.

Costello JP, Olivieri LJ, Su L, Krieger A, Alfares F, Thabit O, Marshall MB, Yoo SJ, Kim PC, Jonas RA, Nath DS. 2015. Incorporating threedimensional printing into a simulation-based congenital heart disease and critical care training curriculum for resident physicians. Congenit Heart Dis 10:185-190.

Dong Y, Suri HS, Cook DA, Kashani KB, Mullon JJ, Enders FT, Rubin O, Ziv A, Dunn WF 2010. Simulation-based objective assessment discerns clinical proficiency in central line placement: A construct validation. Chest 137:10501056.

Dugo M, Brisotto E, Pasi A, Mangino M, Puggia R, Gatti P, Brunello A, Virgilio B, Caberlotto A, Zagatti R, Toffolo K, Palminteri G, Rizzolo M, Mastrosimone S, Maresca MC. 2014. Ultrasound-guided renal biopsy: Evaluation of different models of biological simulator. G Ital Nefrol 31:4.

Gerstenmaier JF, McCarthy CJ, Brophy DP, Cantwell CP. 2013. Evaluation of the particulate concentration in a gelatin-based phantom for sonographically guided lesion biopsy. J Ultrasound Med 32:1471-1475.
Gerstle TL, Ibrahim AM, Kim PS, Lee BT, Lin SJ. 2014. A plastic surgery application in evolution: Three-dimensional printing. Plast Reconstr Surg 133: 446-451.

Groarke PJ, Kelly JC, Flanagan E, Stephens MM. 2015. The forgotten foot An assessment of foot and ankle radiograph pathology in final year medical students. Surgeon (in press; doi: 10.1016/j.surge.2014.03.011).

Groth C, Kravitz ND, Jones PE, Graham JW, Redmond WR. 2014. Threedimensional printing technology. J Clin Orthod 48:475-485.

Hall MK, Mirjalili SA, Moore CL, Rizzolo LJ. 2015. The student's dilemma, liver edition: Incorporating the sonographer's language into clinical anatomy education. Anat Sci Educ 8:283-288.

Johnson SJ, Hunt CM, Woolnough HM, Crawshaw M, Kilkenny C, Gould DA, England A, Sinha A, Villard PF 2012. Virtual reality, ultrasound-guided liver biopsy simulator: Development and performance discrimination. $\mathrm{Br} \mathrm{J}$ Radiol 85:555-561.

Khan IS, Kelly PD, Singer RJ 2014. Prototyping of cerebral vasculature physical models. Surg Neurol Int 5:11.

Li J, Nie L, Li Z, Lin L, Tang L, Ouyang J. 2012. Maximizing modern distribution of complex anatomical spatial information: 3D reconstruction and rapid prototype production of anatomical corrosion casts of human specimens. Anat Sci Educ 5:330-339.

McGaghie WC, Draycott TJ, Dunn WF, Lopez CM, Stefanidis D 2011. Evaluating the impact of simulation on translational patient outcomes. Simul Healthc 6:S42-S47.

McMenamin PG, Quayle MR, McHenry CR, Adams JW. 2014. The production of anatomical teaching resources using three-dimensional (3D) printing technology. Anat Sci Educ 7:479-486.

Miller SF, Sanz-Guerrero J, Dodde RE, Johnson DD, Bhawuk A, Gurm HS, Shih AJ. 2013. A pulsatile blood vessel system for a femoral arterial access clinical simulation model. Med Eng Phys 35:1518-1524.

Mitsuhashi N, Fujieda K, Tamura T, Kawamoto S, Takagi T, Okubo K. 2009. BodyParts3D: 3D structure database for anatomical concepts. Nucleic Acids Res 37:D782-D785.

Mix AW, Giacomin AJ 2011. Standardized polymer durometry. J Test Eval 39. 696-705.

Moscova M, Bryce DA, Sindhusake D, Young N. 2015. Integration of medical imaging including ultrasound into a new clinical anatomy curriculum. Anat Sci Educ (in press; doi: 10.1002/ase.1481).

Motoike HK, O'Kane RL, Lenchner E, Haspel C. 2009. Clay modeling as a method to learn human muscles: A community college study. Anat Sci Educ 2: 19-23.

Petrinec K, Savitsky E, Hein C. 2011. Patient-specific cases for an ultrasound training simulator. Stud Health Technol Inform 163:447-453.

Preece D, Williams SB, Lam R, Weller R. 2013. "Let's get physical": Advantages of a physical model over 3D computer models and textbooks in learning imaging anatomy. Anat Sci Educ 6:216-224.

Rengier F, Mehndiratta A, von Tengg-Kobligk H, Zechmann CM, Unterhinninghofen R, Kauczor HU, Giesel FL. 2010. 3D printing based on imaging data: Review of medical applications. Int J Comput Assist Radiol Surg 5:335-341.

Roche ET, Wohlfarth R, Overvelde JT, Vasilyev NV, Pigula FA, Mooney DJ, Bertoldi K, Walsh CJ. 2014. A bioinspired soft actuated material. Adv Mater 26:1200-1206.

Rose AS, Kimbell JS, Webster CE, Harrysson OL, Formeister EJ, Buchman CA. 2015. Multi-material 3D models for temporal bone surgical simulation. Ann Otol Rhinol Laryngol (in press; doi: 10.1177/0003489415570937).

Seto AH, Abu-Fadel MS, Sparling JM, Zacharias SJ, Daly TS, Harrison AT, Suh WM, Vera JA, Aston CE, Winters RJ, Patel PM, Hennebry TA, Kern MJ. 2010. Real-time ultrasound guidance facilitates femoral arterial access and reduces vascular complications: FAUST (femoral arterial access with ultrasound trial). JACC Cardiovasc Interv 3:751-758.

Torres K, Staśkiewicz G, Śnieżyński M, Drop A, Maciejewski R. 2011. Application of rapid prototyping techniques for modelling of anatomical structures in medical training and education. Folia Morphol (Warsz) 70:1-4.

Turney BW. 2014. A new model with an anatomically accurate human renal collecting system for training in fluoroscopy-guided percutaneous nephrolithotomy access. J Endourol 28:360-363.

Vaccarezza M, Papa V. 2015. 3D printing: A valuable resource in human anatomy education. Anat Sci Int 90:64-65.

Vieira SL, Pavan TZ. Junior JE. Carneiro AA 2013. Paraffin-gel tissuemimicking material for ultrasound-guided needle biopsy phantom. Ultrasound Med Biol 39:2477-2484.

Vidal FP, Villard PF, Holbrey R, John NW, Bello F, Bulpitt A, Gould DA. 2009. Developing an immersive ultrasound guided needle puncture simulator. Stud Health Technol Inform 142:398-400.

Villard PF, Vidal FP, ap Cenydd L, Holbrey R, Pisharody S, Johnson S, Bulpitt A, John NW, Bello F, Gould D. 2014. Interventional radiology virtual simulator for liver biopsy. Int J Comput Assist Radiol Surg 9:255-267.

Watson RA. 2014. A low-cost surgical application of additive fabrication.

J Surg Educ 71:14-17. 